\title{
Philosophiques
}

\section{Tarski et la suppositio materialis}

\section{Claude Panaccio}

Volume 31, numéro 2, automne 2004

URI : https://id.erudit.org/iderudit/009809ar

DOI : https://doi.org/10.7202/009809ar

Aller au sommaire du numéro

\section{Éditeur(s)}

Société de philosophie du Québec

\section{ISSN}

0316-2923 (imprimé)

1492-1391 (numérique)

Découvrir la revue

\section{Citer cet article}

Panaccio, C. (2004). Tarski et la suppositio materialis. Philosophiques, 31(2), 295-309. https://doi.org/10.7202/009809ar

\section{Résumé de l'article}

Dans son article de 1944, "The Semantic Conception of Truth and the Foundations of Semantics ", Alfred Tarski réfère en propres termes à la notion médiévale de "suppositio materialis ». L'interprétation qu'il en suggère, cependant, est historiquement trompeuse et l'inexactitude historique se double, en l'occurrence, de ce que l'on peut tenir pour une malencontreuse erreur philosophique. Dans « "la neige est blanche" est vraie », Tarski voit l'expression « la neige est blanche » (entre guillemets) comme le nom d'une certaine phrase, alors que les médiévaux y auraient vu plutôt une occurrence de cette phrase elle-même prise dans un usage spécial, la suppositio materialis. L'article montre en quoi les deux approches diffèrent et soutient que la théorie médiévale est philosophiquement préférable, en ce que : 1) elle est descriptivement plus adéquate en ce qui concerne le fonctionnement réel des langues naturelles ; 2) elle est plus appropriée même pour les langages construits qu'elle rend plus fonctionnels et plus intelligibles ; 3) elle repose sur l'identification d'un phénomène important dont la généralité échappe aux sémantiques d'inspiration tarskienne, celui de la dualité de principe entre l'extension d'un terme pris en lui-même et celle qu'il reçoit dans un contexte propositionnel donné. 


\title{
Tarski et la suppositio materialis
}

\author{
CLAUDE PANACCIO \\ Université du Québec à Montréal \\ panaccio.claude@uqam.ca
}

\begin{abstract}
RÉSUMÉ. - Dans son article de 1944, «The Semantic Conception of Truth and the Foundations of Semantics », Alfred Tarski réfère en propres termes à la notion médiévale de «suppositio materialis». L'interprétation qu'il en suggère, cependant, est historiquement trompeuse et l'inexactitude historique se double, en l'occurrence, de ce que l'on peut tenir pour une malencontreuse erreur philosophique. Dans « "la neige est blanche" est vraie», Tarski voit l'expression «la neige est blanche» (entre guillemets) comme le nom d'une certaine phrase, alors que les médiévaux y auraient vu plutôt une occurrence de cette phrase elle-même prise dans un usage spécial, la suppositio materialis. L'article montre en quoi les deux approches diffèrent et soutient que la théorie médiévale est philosophiquement préférable, en ce que : 1) elle est descriptivement plus adéquate en ce qui concerne le fonctionnement réel des langues naturelles; 2) elle est plus appropriée même pour les langages construits qu'elle rend plus fonctionnels et plus intelligibles; 3 ) elle repose sur l'identification d'un phénomène important dont la généralité échappe aux sémantiques d'inspiration tarskienne, celui de la dualité de principe entre l'extension d'un terme pris en lui-même et celle qu'il reçoit dans un contexte propositionnel donné.
\end{abstract}

\begin{abstract}
In his 1944 paper "The Semantic Conception of Truth and the Foundations of Semantics", Alfred Tarski refers in so many words to the medieval idea of "suppositio materialis". The interpretation he suggests for it, however, is historically misleading, and this historical inaccuracy yields in this case what can be taken to be an unfortunate philosophical mistake. In "snow is white' is true ", Tarski sees the phrase "snow is white" (between quotation marks) as the name of a certain sentence, while the medieval philosophers would have seen it rather as an occurrence of that very sentence, but taken in a special use, the suppositio materialis. The paper shows how these two approaches differ exactly and argues that the medieval theory is philosophically preferable in that (1) it is descriptively more adequate with respect to natural languages, (2) it is more appropriate even for artificial languages, which it renders both more effective and more intelligible, and (3) it rests upon the identification of an important phenomenon, the generality of which is missed by the Tarskian type semantics, namely the duality of principle between the extension of a term in itself and the extension it receives within a given propositional context.
\end{abstract}

Dans son célébrissime article de 1944, «The Semantic Conception of Truth and the Foundations of Semantics ", Alfred Tarski évoque au passage - et en propres termes - la notion médiévale de "suppositio materialis ${ }^{1}$ ". L'interprétation qu'il en suggère, cependant, est historiquement trompeuse et l'inexactitude historique, en l'occurrence, se double de ce qui me semble être une erreur philosophique. Ou à tout le moins une malencontreuse cécité à

1. J'utiliserai ici, pour les citations tirées de cet article, la traduction française de 1974, réalisée sous la direction de Gilles Gaston Granger, mais avec quelques amendements mineurs. 
l'endroit d'une possibilité théorique qui eût certainement été pertinente pour son propos. C'est ce que je voudrais montrer ici : en ratant ce qui fait l'intérêt de la notion médiévale de suppositio materialis, Tarski est passé à côté d'une idée théorique majeure pour la sémantique philosophique. Et l'omission, malheureusement, s'est perpétuée dans la philosophie du langage dominante de la deuxième moitié $\mathrm{du} \mathrm{xx}^{\mathrm{e}}$ siècle.

\section{Deux théories}

Le passage de Tarski auquel je pense se situe vers le début de l'article, à la section 4 plus précisément, intitulée «Un critère d'adéquation matérielle de la définition ». Le projet de l'auteur, comme on le sait, était de proposer une définition de la notion de vérité pour certains langages ${ }^{2}$. L'idée informelle de vérité qui l'intéressait, ainsi qu'il nous le dit lui-même dans les lignes qui précèdent immédiatement celles qui nous retiendront ici, était l'idée aristotélicienne de vérité-correspondance, selon laquelle, intuitivement, une phrase est vraie si elle dit les choses telles qu'elles sont. Cette formulation, bien sûr, est très imprécise et Tarski, à juste titre, était en quête d'une définition beaucoup plus rigoureuse, à laquelle il impose d'entrée de jeu deux critères d'adéquation : un critère d'adéquation matérielle et un critère d'adéquation formelle. Le second d'entre eux revient à exiger que la définition recherchée soit elle-même formulée dans un langage rigoureusement spécifié, c'est-à-dire au bout du compte un langage formel artificiellement construit. Mais je laisserai ici cet aspect de côté pour me concentrer sur le seul critère d'adéquation matérielle.

Ce critère, extrêmement connu, est le suivant : la définition de la vérité pour un langage donné doit impliquer toutes les équivalences $T$ relatives aux phrases de ce langage, une équivalence $T$ étant elle-même, dans ce vocabulaire, une phrase ayant la forme :

- la phrase X est vraie si et seulement si $p$,

où l'on a à la place «X» le nom ou la description d'une certaine phrase du langage objet, et à la place " $p$ » une occurrence de cette phrase elle-même, ou d'une autre qui lui soit exactement équivalente. L'exemple paradigmatique donné par Tarski est familier — et cher aux Québécois —, c'est celui de la phrase «la neige est blanche» ("snow is white» dans l'original anglais) :

Commençons, [dit Tarski,] par un exemple concret. Prenons la phrase «la neige est blanche». Nous nous demandons sous quelles conditions cette phrase est vraie ou fausse. Il semble clair que si nous nous basons sur la conception classique de la vérité, nous dirons que la phrase est vraie si la neige est blanche et fausse si la neige n'est pas blanche. Par conséquent, si notre définition de la vérité doit être conforme à notre conception de celle-ci, elle doit impliquer

2. Ce projet avait été poursuivi plus en détail dans le grand article de Tarski, «Le concept de vérité dans les langages formalisés ", originalement produit en polonais en 1933 et paru en allemand en 1936, par rapport auquel le texte de 1944 constitue une version vulgarisée. 
l'équivalence suivante : La phrase «la neige est blanche» est vraie si et seulement si la neige est blanche ${ }^{3}$.

Et la même chose doit valoir pour toutes les phrases du langage objet qui sont susceptibles d'être vraies ou fausses. Pour chacune d'entre elles, on doit pouvoir tirer de la définition de la vérité une équivalence de la forme : " $p$ " est vraie si et seulement si $q$, où " $q$ » est exactement synonyme de " $p$ ». Tel est donc le critère d'adéquation matérielle de Tarski. Ce n'est pas encore, rappelons-le, la définition elle-même (comme on le dit parfois), celle-ci, beaucoup plus complexe, étant construite ensuite à partir de la notion de "satisfaction». Mais on ne s'intéressera ici qu'au critère en question.

Où est-il, là, question de «suppositio materialis » ? Continuons tout simplement la lecture du texte de Tarski :

Qu'il me soit permis de remarquer, [ajoute-t-il,] que l'expression «la neige est blanche » se trouve prise, à gauche du signe de l'équivalence, entre guillemets, et à droite sans guillemets. Nous avons à droite la phrase même et à gauche le nom de cette phrase. En employant la terminologie logique médiévale nous pouvons aussi dire que l'expression «la neige est blanche » est prise à droite en supposition formelle (suppositio formalis) et à gauche en supposition matérielle (suppositio materialis) ${ }^{4}$.

Pourquoi devons-nous avoir à gauche le nom de la phrase et non pas la phrase même? Cela saute aux yeux, dit Tarski, et requiert à peine une explication. Mais il en donne tout de même deux raisons. La première est que si on avait là la phrase elle-même plutôt que son nom, le résultat serait agrammatical : le schéma «X est vrai» n'accepte pas une phrase complète en position $\mathrm{X}$. La deuxième raison est encore plus saisissante :

Et, en deuxième lieu, [écrit Tarski,] les conventions les plus fondamentales qui règlent l'usage de tout langage exigent que dans chaque expression que nous énonçons au sujet d'un objet le nom de celui-ci soit employé et non cet objet même. Par conséquent, si nous désirons dire quelque chose au sujet d'une phrase, qu'elle est vraie, par exemple, nous devons employer son nom et non elle-même 5 .

Ce passage soulève un certain nombre de problèmes, que je veux maintenant passer en revue.

Commençons par l'inexactitude historique. Je ne parle pas ici de l'emploi de «suppositio formalis». L'expression, quoique rare au Moyen Âge on ne la trouve, par exemple, ni dans le célèbre traité de logique de Pierre d'Espagne, le Tractatus, au XIII ${ }^{\text {e }}$ siècle ni dans la Somme de logique de

3. Tarski, 1944, trad. Granger, 1974, p. 271 (NB : Je remplace partout le terme "proposition" utilisé par les traducteurs français, par "phrase ", qui rend mieux le «sentence» de l'original anglais).

4. Ibid., pp. 271-272.

5. Ibid., p. 272. 


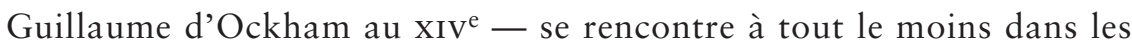
Introductiones in logicam de l'Anglais Guillaume de Sherwood, où elle est bel et bien opposée à la suppositio materialis ${ }^{6}$. C'est plutôt la notion même de suppositio materialis - très courante, elle, chez les logiciens médiévaux — qui fait problème. Selon Tarski, dans les lignes que l'on vient de lire, dire qu'une expression E est prise en suppositio materialis dans un certain contexte est une façon de dire qu'apparaît dans ce contexte un nom de l'expression E, formé d'une façon spéciale à partir de cette expression elle-même et d'une marque conventionnelle comme les guillemets. Le mot "Tarski» placé entre guillemets, par exemple, deviendrait par le fait même une nouvelle expression, qui serait non plus le nom de Tarski, comme l'était le terme original, mais le nom de son nom.

L'inexactitude historique, ici, est la suivante. Les médiévaux qui utilisaient la terminologie en question n'auraient jamais dit que l'expression prise en suppositio materialis est le nom d'une autre expression. Il s'agissait plutôt à leurs yeux d'une occurrence de cette expression elle-même, mais prise dans un usage spécial. Voyez ce qu'en dit Guillaume d'Ockham dans la Somme de logique : Ainsi, dans "homme est un nom", il est évident que "homme" suppose pour lui-même ${ }^{7}$ ». Les médiévaux n'acceptaient tout simplement pas ce que Tarski tient pour l'une des «conventions fondamentales» de tout langage, à savoir que si l'on veut parler d'un objet, il faut employer le nom de cet objet et jamais l'objet lui-même. La doctrine médiévale de la suppositio materialis est en contradiction directe avec ce principe. Ce qu'elle nous dit, justement, c'est que certains objets - les objets linguistiques notamment peuvent être utilisés parfois pour parler d'eux-mêmes. Guillaume d'Ockham, par exemple, admet explicitement au début de la Somme de logique qu'une phrase peut — contrairement à ce que dit Tarski — figurer elle-même en position de sujet : dans " "l'homme est un animal" est vrai ", explique-t-il, c'est la phrase "l'homme est un animal» elle-même qui sert de sujet ${ }^{8}$. L'erreur de Tarski est de croire que la théorie médiévale de la suppositio materialis illustre un principe qu'il juge fondamental pour l'usage linguistique, alors que cette théorie, en fait, est incompatible avec le principe en question.

Cela, certes, n'aurait guère d'importance en soi. Tarski, après tout, est un grand logicien et l'on aurait mauvaise grâce à lui reprocher de ne pas être aussi un bon médiéviste! Mais ce qu'il y a d'intéressant dans le cas qui nous occupe, c'est la divergence philosophique qui se révèle ainsi entre Tarski et les médiévaux. Et cette divergence, elle, est loin d'être sans conséquence.

Il suit, d'abord, du principe de Tarski selon lequel un objet donné, fûtil linguistique, ne peut jamais être employé dans une phrase pour y tenir lieu

6. Voir Guillaume de Sherwood, Introductiones in logicam, 5.1.2, éd. Lohr, 1983, p. 266.

7. Guillaume d'Ockham, Somme de logique, I, 64, trad. Biard, 1993, p. 202 (les italiques sont de moi). Pour un bon aperçu général de la théorie médiévale de la suppositio materialis, voir Rosier-Catach, 2000.

8. Ibid., I, 2, trad. Biard, 1993, p. 8. 
de lui-même, que la sémantique d'un langage L quelconque requiert toujours de disposer d'un nom distinct pour chaque expression de L, et en particulier, pour ce qui est de la théorie de la vérité dans L, d'un nom distinct pour chaque phrase de L. Comme certains langages autorisent, potentiellement, une infinité de phrases grammaticales, la sémantique de ces langages devrait pouvoir disposer d'une infinité de noms de phrases. Et si l'on voulait envisager, en plus, de produire la sémantique d'un langage donné dans ce langage même (ce que Tarski ne fait pas, il faut le dire), on aurait ipso facto une nouvelle démultiplication des noms à l'infini, puisque chaque nouveau nom $-\mathrm{y}$ compris les noms de phrases et les noms de noms - aurait à son tour besoin d'être nommé. Cet extraordinaire enrichissement du vocabulaire, sans doute, n'est pas dirimant en lui-même, puisque chaque nouveau nom est supposé être construit à l'aide d'un procédé bien défini à partir des expressions déjà introduites dans la langue en question. Mais il n'en est pas moins surprenant, et fort peu économique. Dans la théorie médiévale de la suppositio, en revanche, la phrase en supposition matérielle est construite comme n'importe quelle autre occurrence de la même phrase, c'est-à-dire en combinant ses composantes de façon appropriée, et aucun nouveau nom n'est ajouté. On admet, tout simplement, qu'il y a pour chaque expression bien construite de la langue certains usages spéciaux, qui modifient notamment les conditions de vérité des phrases où ces expressions apparaissent. On peut envisager de multiplier ces usages et de complexifier en conséquence la théorie de la suppositio (en admettant, par exemple, une suppositio spéciale pour les termes écrits, une autre pour les termes oraux, une autre encore pour les mots écrits ou oraux indifféremment, et ainsi de suite). Mais on n'aura jamais au bout du compte qu'un nombre très limité de telles variétés de suppositio, et qui n'entraîneront par elles-mêmes aucune démultiplication spéciale des expressions admissibles dans le langage.

Les différences entre les deux approches, cependant, sont encore plus profondes et ne tiennent pas seulement au nombre d'unités qu'elles admettent dans le vocabulaire. La théorie tarskienne de la guillemetisation repose sur deux principes tenus pour allant de soi :

(1) aucune expression linguistique ne peut apparaître en position de sujet grammatical à moins d'être un nom (ou plus généralement : la catégorie grammaticale d'une expression linguistique en commande de façon restrictive tous les usages);

(2) aucune expression linguistique ne peut être employée de façon suiréférentielle (à moins, bien sûr, que cela ne découle de sa signification habituelle, comme c'est le cas, par exemple, du mot "nom»).

La doctrine médiévale, elle, rejette ces deux principes. Son idée de base, étrangère à la sémantique contemporaine, est qu'une expression linguistique peut avoir deux sortes bien distinctes de propriétés sémantiques. Elle a une signification d'abord, qui ne varie pas selon les contextes propositionnels, et 
qui détermine notamment la catégorie grammaticale à laquelle le terme appartient. Une expression linguistique quelconque, dans cette optique, ne devient pas un nom en jouant tel ou tel rôle dans une phrase. Elle est un nom ou elle n'en est pas un avant même de figurer dans des phrases. La catégorie grammaticale d'une expression linguistique, en d'autres mots, est associée au type — c'est-à-dire à toutes les occurrences indifféremment — et non pas à telles ou telles occurrences en particulier à l'intérieur des phrases ${ }^{9}$. La deuxième sorte de propriété sémantique, au contraire, est celle que l'expression linguistique reçoit lorsqu'elle est en position de sujet ou de prédicat dans le contexte d'une phrase. C'est la suppositio. Cela revient à dire que l'expression en question tient lieu dans cette phrase de certains objets du monde, qu'elle a, si l'on veut, une fonction référentielle dans la phrase.

Dans le cas le plus habituel, il y a une coïncidence assez grande entre la signification prépropositionnelle et la suppositio. Pour Guillaume d'Ockham, par exemple, un nom propre comme "Cicéron" signifie par institution conventionnelle l'individu réel Cicéron et lorsqu'il est pris dans son usage normal tient lieu de — ou "suppose pour " — ce même individu. C'est à cause de cette coïncidence, sans nul doute, que de nombreux théoriciens du langage - et, en particulier, de nombreux théoriciens contemporains — n'ont pas senti le besoin de distinguer très nettement entre la propriété sémantique prépropositionnelle et la fonction référentielle acquise en contexte propositionnel. Mais si cette distinction était tenue pour nécessaire par les médiévaux, c'est qu'il y a malgré tout, remarquaient-ils, de fréquents écarts entre la signification prépropositionnelle et la fonction référentielle en contexte propositionnel. Ces écarts, selon leur conception, peuvent tenir à différents facteurs. Le temps du verbe, par exemple, en est un; j'y reviendrai. Mais l'écart le plus spectaculaire, dans cette optique, est celui, précisément, qui concerne la suppositio materialis. Une expression quelconque, pensaient-ils, peut être utilisée dans certains contextes propositionnels pour se représenter elle-même (ou pour représenter une forme linguistique étroitement apparentée à la sienne, comme le génitif «Ciceronis » peut représenter dans certains contextes le nominatif «Cicero»). Cette possibilité d'un usage spécial suiréférentiel était reconnue pour toutes les expressions linguistiques, quelle que soit leur catégorie grammaticale. Dans " "et" est une conjonction", par exemple, le mot «et» n'est pas devenu un nom, selon la théorie médiévale. Il reste bel et bien une conjonction, cela étant déterminé par sa propriété sémantique prépropositionnelle, mais cette conjonction est maintenant prise, dans ce contexte propositionnel particulier, selon un usage spécial, la suppositio materialis en l'occurrence, et à ce titre se représente elle-même dans ce contexte. La même chose, évidemment, vaut pour les phrases complètes.

9. Il peut arriver certes que deux occurrences homophoniques appartiennent à des catégories grammaticales distinctes - «court» comme adjectif masculin singulier et «court» comme verbe à la troisième personne du présent, par exemple. Mais elles ne seront pas considérées dans ce cas comme des occurrences d'un même type linguistique. 


\section{Avantages et inconvénients}

On a donc bien là deux théories différentes. L'une, celle de Tarski, veut qu'une expression ne puisse jamais tenir lieu d'elle-même et elle recourt à une notation ad hoc - les guillemets - pour expliquer la formation d'une sorte spéciale d'expressions métalinguistiques. L'autre, celle des médiévaux, distingue dans tous les cas les propriétés sémantiques des termes pris en eux-mêmes (toujours associés à leur catégorie grammaticale) et les fonctions référentielles que ces termes peuvent jouer quand ils apparaissent dans des phrases, et explique à partir de là les usages autonymiques. Comment ces deux théories se comparent-elles pour ce qui est des avantages et des inconvénients? La thèse que je voudrais proposer à l'attention bienveillante du lecteur est que l'approche médiévale, au total, fournit une meilleure théorie. J'utiliserai pour le faire voir trois ordres de considérations. Et je discuterai, en fin de texte, une objection intéressante inspirée de Tarski.

2.1 En tout premier lieu, il faut le dire avec insistance, la conception selon laquelle "Socrate» dans " "Socrate" est un nom » ou «la neige est blanche" dans " "la neige est blanche" est vraie" sont des occurrences du nom "Socrate» lui-même ou de la phrase «la neige est blanche » elle-même, mais pris selon un usage spécial (qu'on peut appeler la suppositio materialis), présente, relativement aux langues naturelles en tout cas, une adéquation descriptive que n'a pas prima facie la théorie des noms de noms ou des noms de phrases. Nous avons tous conscience, comme locuteurs, d'employer dans ce genre de cas l'expression elle-même, mais dans un usage spécial. Josette ReyDebove, dans son livre Le métalangage, quoique sympathique à la théorie des noms de noms, rapporte qu'aucun des locuteurs qu'elle a interrogés à ce sujet — même parmi les linguistes — «n'a exprimé le sentiment » d'une dualité lexicale entre les noms et les noms de noms ${ }^{10}$, dualité qui est pourtant requise par l'approche tarskienne. François Recanati, lui, est très affirmatif : «il n'y a aucun doute, écrit-il dans un article récent, que le langage naturel permette d'utiliser un mot pour référer à ce mot lui-même ${ }^{11}$. » Et il le dit justement pour contester que la théorie des noms de noms soit "descriptivement adéquate» pour rendre compte de la façon dont les langues naturelles fonctionnent. John Searle encore, pour ne prendre qu'un seul autre exemple, commentant dans Speech Acts le passage même de Tarski dont nous sommes partis - celui qui dit que c'est une «convention fondamentale» de tout langage que l'on n'utilise jamais dans le langage l'objet même auquel on veut référer - écrit, un peu brutalement, à ce sujet :

La seule réponse qu'on puisse faire à cela, c'est qu'il n'y a pas de convention fondamentale de ce genre. Certains fragments de discours, qu'ils se présentent sous une forme orale ou visuelle, peuvent très bien apparaître dans le discours

10. Rey-Debove, 1997, p. 145.

11. Recanati, 2000, p. 368 (ma traduction). 
en tant qu'objet de discours. Un ornithologue par exemple peut très bien dire "le cri du geai de Californie est... ». Et ce qui complète la phrase est un cri, et non le nom propre d'un $\mathrm{cri}^{12}$.

Mais si Searle, Recanati, et tous les locuteurs interrogés par Rey-Debove ont raison sur ce point, cela suggère fortement qu'il faut distinguer, comme le faisaient les médiévaux, entre les propriétés sémantiques qui sont constitutives du mot pris en lui-même et les fonctions référentielles diverses qu'il peut avoir en contexte.

Cela permet, du reste, de rendre compte de nombreux phénomènes tout à fait courants. Il est clair, par exemple, que nous pouvons mentionner dans une langue donnée une expression linguistique appartenant à une autre langue. Je peux dire en français "wheather est un mot anglais". Ce qui montre, dans un tel cas, que le sujet de cette phrase n'est pas le nom français du mot anglais, mais le mot anglais lui-même, c'est qu'il s'y trouve un phonème (exprimé par «th») qui n'est pas un phonème du français. On aurait pu certes prononcer la phrase à la française : "ouèdeure est un mot anglais ". Mais il est suffisant pour ma thèse que cela ne soit pas obligatoire. L'important est qu'il est admissible d'introduire dans une phrase orale française des phonèmes d'une autre langue quand on veut parler des expressions de cette langue. Cela est particulièrement net quand il s'agit d'évoquer la prononciation étrangère elle-même. Je peux dire «les Anglais prononcent wheather et non ouèdeure" ou, selon un exemple proposé par Josette Rey-Debove, «les Allemands disent Geist». La raison évidente pour laquelle ces phrases sont correctes en français même si elles contiennent au moins un phonème qui n'appartient pas au français, c'est qu'il est permis d'utiliser dans une langue des mots d'une autre langue lorsqu'ils sont pris en supposition matérielle. La chose vaut même pour les expressions qui ne sont d'aucune langue, comme dans "Jean a fait waou» ou - un exemple courant au Moyen Âge — «buba a quatre lettres », ou comme dans l'exemple de Searle, de l'ornithologue qui imite le cri du geai.

L'adéquation supérieure de la théorie de la suppositio materialis par rapport à l'approche tarskienne se montre encore à ceci qu'elle donne une réponse toute naturelle, sur le plan descriptif, à la fameuse énigme d'Anscombe : il est impossible que l'on me dise le nom de quiconque, car si l'on me dit «le nom de ce monsieur est "Smith" ", ce que j'entends, c'est le nom de son nom, mais non pas son nom lui-même ${ }^{13}$. Pour la théorie de la suppositio, la solution coule de source : cela est un faux problème, qui ne se pose que pour la théorie des noms de noms, car ce que j'entends en fait, c'est bien le nom de la personne, mais pris en suppositio materialis.

2.2 Deuxième ordre d'arguments : même un langage construit fonctionnera mieux et sera plus intelligible si ses règles permettent dans certaines situations l'usage des expressions linguistiques pour se représenter elles-mêmes. 
On évitera ainsi, d'abord, ce lexique métalinguistique infini qui serait rendu nécessaire si chaque expression d'un langage capable d'engendrer une infinité de phrases devait avoir un nom distinct dans le métalangage. Et l'on évitera surtout l'étrange démultiplication ironiquement évoquée par John Searle dans Speech Acts :

Philosophes et logiciens affirment généralement que dans un cas du type de 2 [ «Socrate» a sept lettres], ce n'est pas le mot «Socrate» qui apparait mais bien un mot tout à fait autre qui est le nom propre du mot [...] De ce point de vue, le premier mot de 2 n'est pas, comme vous pourriez le penser : "Socrate", mais « «Socrate»". Et, ce qui est assez difficile à saisir, le mot que je viens d'écrire n'est pas : « «Socrate » ", mais « « «Socrate » " ", mot tout à fait différent, qui est à son tour nom propre du nom propre d'un nom propre, à savoir 《« « «Socrate»»»». Et ainsi de suite dans la hiérarchie des noms de noms.

Pour moi, ce point de vue est absurde ${ }^{14}[\ldots]$

L'inconvénient, à vrai dire, n'est pas seulement que cette démultiplication par la réitération des guillemets engendre un méli-mélo inextricable, mais aussi que l'usage même des guillemets pour créer des noms de noms ou des noms de phrases soulève pour l'approche tarskienne une difficulté théorique assez curieuse : quel est au juste, dans ce genre de cas, l'élément qui est placé entre guillemets? Concernant les noms de phrases, par exemple, Tarski nous a expliqué pour justifier sa position que dans un schéma de forme $X$ est $F$ (comme : X est vrai), on ne peut avoir une phrase complète en position X, parce que le résultat ainsi obtenu serait agrammatical. Le même argument, cependant, vaudrait tout autant pour les contextes de forme " $X$ » est $F$. L'argument de Tarski présuppose en effet que l'on ne peut utiliser une phrase qu'avec une certaine fonction grammaticale, une fonction phrastique, disons. Cela revient à dire, si on le prend au sérieux, que l'on ne peut combiner de façon grammaticalement correcte une phrase complète avec autre chose que par le recours à des connecteurs phrastiques comme "et ", "ou ", "si », "parce que ", "que ", etc., comme dans «la neige est blanche et l'herbe est verte» ou «Tarski pense que la neige est blanche». Mais si ce principe interdit de placer une occurrence de phrase dans un contexte de forme _ est $F$, il interdira aussi, exactement pour la même raison, de placer une occurrence de phrase dans un contexte de forme "_" est $F$, qui ne comporte pas plus que l'autre de connecteur phrastique ou propositionnel. Les guillemets tarskiens n'expriment pas une fonction ordinaire et a fortiori ne sont pas des connecteurs phrastiques ou propositionnels.

Considérons un cas plus simple, celui d'un nom comme "Cicéron". Pour former à la Tarski le nom de ce nom, il faut prendre une occurrence du nom lui-même et la placer entre guillemets. Mais que s'est-il alors passé ? Les guillemets ne fonctionnent pas à la façon d'une expression comme «le père 
de _". Quand on forme l'expression complexe «le père de Cicéron », le nom "Cicéron" qui y figure nomme bien Cicéron lui-même et le résultat obtenu est fonction de Cicéron : l'expression "le père de Cicéron » désigne le père de Cicéron. Mais s'il en allait ainsi pour les guillemets, il faudrait que le nom "Cicéron » dans " "Cicéron" " nomme encore Cicéron lui-même comme d'habitude, et que le résultat obtenu soit fonction de Cicéron, ce qui n'est pas le cas. Il n'est pas vrai, en particulier, que l'expression "Cicéron" " (avec les guillemets) équivaille à l'expression "le nom de Cicéron " puisque Cicéron peut avoir en fait plusieurs noms. Admettons qu'il s'appelle aussi Tullius, par exemple : les phrases "le nom de Cicéron commence par un "t" " et " "Cicéron" commence par un " $\mathrm{t}$ " " n'auraient pas nécessairement, dans ce cas, la même valeur de vérité.

On serait tenté sans doute de dire que le mot qui est inséré entre les guillemets est le nom "Cicéron" lui-même, pris dans un usage spécial en vertu duquel il ne représente plus l'individu Cicéron, mais se représente luimême comme mot. Cette solution, cependant, est précisément celle que l'approche tarskienne rejette! Il y a quelque chose d'étrange, bref, à dire que le nom lui-même ou la phrase elle-même sont mis entre guillemets si l'on n'accepte pas par ailleurs la légitimité d'un usage autonyme spécial des noms et des phrases. L'interdiction tarskienne d'utiliser une expression linguistique pour se représenter elle-même conduit de la sorte à une énigme difficile qui ne semble pas admettre, dans ce cadre théorique, de solution naturelle.

La doctrine de la suppositio materialis est en bien meilleure posture à cet égard. Il est vrai que les guillemets n'existaient pas au Moyen Âge et que les logiciens de l'époque, en conséquence, n'en ont jamais traité. Mais ce que la théorie peut - et doit — dire à leur sujet, c'est tout simplement ceci : les guillemets dans une phrase comme «homme» est un nom sont des marqueurs de supposition matérielle ${ }^{15}$. Ce ne sont pas des foncteurs permettant de former de nouveaux noms, mais des syncatégorèmes indiquant que l'expression ainsi encadrée, qu'il s'agisse d'un nom, d'une phrase ou de quoi que ce soit d'autre, est prise en supposition matérielle. Il n'y a là aucun mystère.

Une objection de principe à l'approche par la suppositio materialis pour les langages construits pourrait être que la variation de la supposition, dans ce genre de cas, engendre des ambiguités systématiques, ce qu'un langage construit ne devrait pas permettre, ou le moins possible ${ }^{16}$. Mais c'est un problème qui est facile à régler : il suffit de disposer d'un marqueur de supposition matérielle,

15. Il est à noter d'ailleurs que si le Moyen Âge ne connaissait pas les guillemets, il a néanmoins fini par introduire dans le latin de l'École un autre marqueur de supposition matérielle, sous la forme d'un article spécial emprunté au français : ly (ou li). "Homme» est un nom, dans cette notation, se disait : ly homo est nomen.

16. Guillaume d'Ockham, par exemple, admet que le terme «Socrate» dans une phrase comme " "Socrate" est un nom" peut avoir soit la supposition personnelle (la phrase signifie alors que l'individu Socrate est un nom), soit la supposition matérielle (et la phrase signifie que le mot «Socrate» est un nom). Cf. Somme de logique, I, 65. 
comme les guillemets (ou l'article médiéval «ly»). On aura alors une construction beaucoup plus économique et beaucoup plus fonctionnelle que s'il nous fallait démultiplier le lexique à l'infini ou introduire un foncteur spécial. Dans un langage autorisant la supposition matérielle, on peut à cet égard se contenter d'une règle très simple : chaque expression peut être employée pour se représenter elle-même (ou, en tout cas, d'un nombre très restreint de règles de ce genre ${ }^{17}$ ), plus un marqueur de supposition matérielle. On gagne alors sur tous les tableaux, avec un langage plus simple sur le plan lexical, mais qui évite tout aussi bien les ambiguïtés (mieux même : pensez au passage de Searle sur «Socrate» cité ci-dessus!).

2.3 Un troisième ordre de considérations, maintenant, ouvre à la réflexion de nouvelles perspectives, qui sont, à mes yeux, les plus importantes : dans la théorie médiévale, la suppositio materialis n'est qu'un cas particulier d'un phénomène beaucoup plus général, celui de la variation de la fonction référentielle des expressions linguistiques prises en contexte propositionnel, ou, si l'on préfère, de la variation de l'extension des expressions selon les contextes. Le cas de la suppositio materialis est particulièrement spectaculaire, certes, parce qu'il n'y a en général aucune intersection entre l'extension prépropositionnelle d'un terme donné - ce que Guillaume d'Ockham appelle sa signification — et l'extension que le même terme reçoit dans un contexte où il est pris en suppositio materialis. Il n'y a pas d'intersection, par exemple, entre l'extension prépropositionnelle de «cheval » en français (qui correspond à l'ensemble de tous les chevaux) et l'extension contextuelle de "cheval» dans " "cheval" est un nom » ou " "cheval” a six lettres ». Mais il n'en reste pas moins que c'est un cas particulier.

Un autre cas, par exemple, bien connu des médiévaux, où il y a un écart entre l'extension prépropositionnelle et l'extension contextuelle, est celui très courant — où le temps du verbe opère une « restriction ». Considérons la phrase suivante :

(1) Depuis le 11 septembre 2001, les Américains sont tous inquiets.

On ne peut évidemment pas inférer de (1) que George Washington ou Abraham Lincoln sont devenus inquiets depuis le 11 septembre, même si George Washington et Abraham Lincoln appartiennent en principe à l'extension prépropositionnelle du terme "Américain». La raison en est que les indications temporelles fournies par (1) (le complément de temps introduit par «depuis» et le temps présent du verbe principal) restreignent l'extension du terme «Américains» dans cette phrase aux seuls Américains qui existent au moment de l'énonciation. Comparons avec :

(2) En septembre 1863, tous les Américains étaient préoccupés par la Guerre de Sécession.

17. Pour un traitement plus détaillé des règles requises par une théorie de la suppositio materialis, en particulier si elle se veut nominaliste, voir Panaccio et Perini Santos, à paraître, Vivarium, vol. 42. 
On ne peut pas, cette fois, inférer de (2) que George W. Bush et son père - qui pourtant appartiennent, paradigmatiquement, à l'extension prépropositionnelle du terme "Américain " - aient été très préoccupés par la Guerre de Sécession en septembre 1863, pour la bonne raison que les indications temporelles présentes dans (2) restreignent, malgré le quantificateur universel, l'extension contextuelle du terme "Américains" aux seuls Américains qui existaient en septembre 1863.

Bref, il y a des raisons indépendantes pour poser une distinction sémantique entre l'extension prépropositionnelle d'un terme ou d'une expression et l'extension de ce même terme ou de cette même expression (ou de leurs occurrences) en contexte propositionnel, c'est-à-dire, pour parler comme Ockham, entre la signification et la supposition ${ }^{18}$. Des raisons qui n'ont rien à voir avec les usages autonymiques. Ceux-ci, en conséquence, lorsqu'ils sont abordés par le biais de l'idée de suppositio materialis, peuvent apparaître comme un cas particulier d'un phénomène plus large, ce que la théorie des noms de noms, pour sa part, empêche d'apercevoir. La sémantique tarskienne, en d'autres mots, rate ici une généralisation.

Résumons :

a) L'approche par la suppositio est descriptivement plus adéquate que la théorie des métanoms en ce qui concerne le fonctionnement réel des langues naturelles.

b) Même pour les langages construits, elle est aussi plus appropriée, parce que les langages qui autorisent la suppositio materialis seront, toutes choses étant égales par ailleurs, plus fonctionnels et plus intelligibles que les métalangages tarskiens.

c) L'approche par la suppositio repose sur l'identification d'un phénomène tout à fait général qui semblait échapper aux sémantiques d'inspiration tarskienne, celui de la dualité de principe entre l'extension qu'a un terme indépendamment du contexte propositionnel et celle qu'il reçoit dans un contexte donné.

2.4 Je voudrais, pour finir, discuter une objection intéressante que l'on peut adresser à l'idée, centrale pour la théorie de la suppositio materialis, que c'est le même terme (ou, en tout cas, des occurrences du même terme) qui figure dans l'usage normal et dans l'usage autonymique. Cette objection, qui correspond, en fait, au premier argument évoqué par Tarski à l'appui de la théorie des noms de noms (voir ci-dessus p. 297), est développée notamment par Josette Rey-Debove ${ }^{19}$ : le comportement grammatical d'une expression prise

18. Cf. Guillaume d'Ockham, Somme de logique, I, 63 : «Maintenant que nous avons traité de la signification des termes, il nous reste à parler de la supposition, propriété qui ne convient jamais au terme qu'au sein d'une proposition» (trad. Biard, 1993, p. 199).

19. Cf. Rey-Debove, 1997, pp. 64-68. Une objection très semblable est adressée à la théorie ockhamiste de la suppositio materialis par Karger, 1982; voir à ce propos Panaccio et Perini Santos, à paraître, Vivarium, vol. 42. 
en supposition matérielle, remarque-t-elle, est très différent, dans beaucoup de cas, de celui de l'expression à laquelle elle réfère; il doit donc s'agir de deux expressions différentes. Prenons :

(3) Rapidement est un adverbe

$\mathrm{Ou}$

(4) Rapidement a dix lettres

Ces deux phrases sont vraies et leur sujet, dans les deux cas, se prononce ra-pi-de-ment. Mais un adverbe ne peut pas grammaticalement être le sujet d'une phrase. Le sujet de (3) et (4), donc, n'est pas l'adverbe « rapidement ", mais le nom de cet adverbe (étant admis que seul un nom - ou un groupe nominal - peut être le sujet d'une phrase). Et d'autres particularités grammaticales encore sont manifestes. En français, par exemple, quand un nom est employé de manière sui-référentielle, il ne prend pas d'article. Comparez :

(5) chat a quatre lettres

avec

(6) le chat a quatre pattes.

De même, un nom féminin est au masculin quand il est pris en mention et un nom pluriel est grammaticalement traité comme un singulier, ainsi qu'il apparaît dans :

(7) vache est plus court que tortue [et non : plus courte],

et

(8) chevaux est un pluriel [et non : sont un pluriel].

Rey-Debove conclut de là que le terme qui est censé apparaître en supposition matérielle est en réalité un autre mot que celui auquel il réfère : l'un et l'autre n'appartiennent pas toujours à la même catégorie grammaticale, ils n'ont pas nécessairement le même genre ni le même nombre, etc.

Cet argument, cependant, pour ingénieux qu'il soit, présuppose sans la discuter une conception très précise de la catégorisation grammaticale, selon laquelle la catégorie grammaticale d'une expression en commande tous les usages. De telle sorte, par exemple, que si un mot est un adverbe, il ne peut jamais figurer qu'en position adverbiale, et ainsi de suite. Or cette conception ne va pas de soi, loin de là. Le théoricien de la suppositio doit considérer, pour sa part, que la catégorie grammaticale d'une expression n'en commande les usages que lorsque l'expression est prise en supposition normale (celle que les médiévaux appelaient la suppositio personalis) et non lorsqu'elle est prise en suppositio materialis. Mais cette conception restrictive de la catégorisation grammaticale ne comporte, pour autant que je puisse voir, aucun inconvénient notable. Elle n'aboutit pas, en particulier - comme on pourrait le craindre à une grammaire anarchique, parce que toutes les expressions en supposition matérielle se comportent de la même façon sur le plan grammatical : elles 
peuvent toutes être sujet, etc. Il n'est pas nécessaire, en conséquence, de les répartir en diverses catégories grammaticales, et l'on n'a besoin pour ces cas-là que de donner les règles grammaticales qui s'appliquent en général à la supposition matérielle.

Il est important en grammaire, on le sait bien, de distinguer nettement entre la catégorie grammaticale d'une expression (nom, verbe, adverbe, etc.), qui est prépropositionnelle, et la fonction grammaticale que joue l'expression dans une phrase donnée (sujet, complément, etc. ${ }^{20}$. La catégorie, certes, est pertinente pour déterminer les fonctions qu'une expression peut remplir, mais dans l'optique de la théorie de la suppositio, cette pertinence se limite aux usages ordinaires de l'expression (c'est-à-dire aux cas de supposition personnelle), les places grammaticales susceptibles d'être occupées par une expression en supposition matérielle étant régies, elles, par un petit nombre de règles ad hoc. Cela complique un peu la grammaire sans doute, mais simplifie du même coup le lexique de façon radicale tout en donnant à la langue une souplesse remarquable, lui permettant, notamment, d'utiliser en supposition matérielle - et avec les fonctions appropriées - des expressions de n'importe quelle catégorie grammaticale, et même des séquences qui à proprement parler n'appartiennent pas à la langue en question (comme dans " "wheather" est un mot anglais", "buba a quatre lettres» ou «il a fait waou»).

L'objection de Rey-Debove oblige donc à des précisions importantes quant à la portée des catégories grammaticales dans un langage qui autorise le genre de phrases qui nous intéressent, mais pour peu que l'on reconnaisse la distinction qui doit être faite entre les catégories et les fonctions grammaticales, elle n'ébranle pas, tant s'en faut, la doctrine de la suppositio materialis comme on l'a ici comprise.

Je conclus, en un mot, qu'il y a lieu aujourd'hui d'envisager beaucoup plus sérieusement qu'on ne le fait d'habitude d'aborder la théorie du langage à partir de la dualité de principe entre les propriétés prépropositionnelles et les propriétés contextuelles, que ce soit sur le plan sémantique ou sur le plan syntaxique. La question de la suppositio materialis, mal comprise par Tarski, représente à cet égard un cas privilégié, où les avantages de l'approche médiévale ressortent, me semble-t-il, avec une certaine netteté. Mais la vieille théorie de la suppositio, qui reposait justement sur cette distinction, recèle encore, à n'en pas douter, bien d'autres richesses insuffisamment exploitées.

20. Remarquons, au passage, que cette distinction est rigoureusement parallèle sur le plan syntaxique à celle que traçaient les médiévaux sur le plan sémantique entre la signification (prépropositionnelle) et la suppositio (contextuelle). 


\section{Bibliographie}

Anscombe, G. E. M. 1981. Metaphysics and the Philosophy of Mind [Collected Philosophical Papers, vol. II], Oxford, Basil Blackwell.

Biard, Joël. 1993. Guillaume d'Ockham. Somme de logique. Première partie, texte latin et traduction française, Mauvezin, Trans-Europ-Repress, $2^{\mathrm{e}}$ éd. revue ( $1^{\text {re }}$ éd. : 1988$)$.

Granger, Gilles-Gaston et al. 1974. Alfred Tarski. Logique, sémantique, métamathématique, 1923-1944, 2 vol., Paris, Armand Colin.

Guillaume de Sherwood : Voir Lohr, 1983.

Guillaume d'Ockham : Voir Biard, 1993.

Karger, Elizabeth. 1982. «La supposition matérielle comme supposition significative : Paul de Venise, Paul de Pergula ", dans Maierù, 1982, pp. 331-341.

Lohr, Charles H. (avec la collab. de P. Kunze et B. Mussler). 1983. «William of Sherwood, "Introductiones in Logicam". Critical Text ", Traditio, 39 , pp. 219-299.

Maierù, Alfonso, dir. 1982. English Logic in Italy in the 14th and 15th Centuries, Naples, Bibliopolis.

Orenstein, Alex et Petr Kotatko, dir. 2000. Knowledge, Language and Logic, Dordrecht, Kluwer.

Panaccio, Claude et Ernesto Perini Santos, "Guillaume d'Ockham et la suppositio materialis », à paraître, Vivarium, vol. 42.

Recanati, François, 2000, "Opacity and the Attitudes ", dans Orenstein et Kotatko, 2000, pp. 367-406.

Rey-Debove, Josette. 1997. Le métalangage. Étude linguistique du discours sur le langage, Paris, Armand Colin, $2^{\mathrm{e}}$ éd. (1 ${ }^{\mathrm{re}}$ éd. : 1978).

Rosier-Catach, Irène. 2000. «La suppositio materialis et la question de l'autonymie au Moyen Âge ", texte présenté au colloque "Le fait autonymique dans les langues et les discours » (Paris, oct. 2000); publication électronique :

http://www.cavi.univ-paris3.fr/ilpga/autonymie/theme1/rosiercat1/pdf.

Searle, John R. 1969. Speech Acts, Cambridge, Cambridge University Press; Les actes de langage, trad. de l'anglais par H. Pauchard, Paris, Hermann, 1972.

Tarski, Alfred. 1936. "Der Wahrheitsbegriff in den formalisierten Sprachen ", Studia philosophica, 1, pp. 261-405; trad. de l'allemand. : « Le concept de vérité dans les langages formalisés », dans Granger, 1974, vol. I, pp. 157-269.

Tarski, Alfred. 1944. "The Semantic Conception of Truth and the Foundations of Semantics ", Philosophy and Phenomenological Research, 4, pp. 341-376; trad. de l'anglais : "La conception sémantique de la vérité et les fondements de la sémantique », dans Granger, 1974, vol. II, pp. 265-305. 